\title{
Congenital absence of the inferior vena cava
}

\author{
Anthony M.-H. Ho, MD, FRCPC, FCCP • Glenio B. Mizubuti, MSc, MD $(1) \cdot$ \\ Andrew D. Chung, MD, FRCPC
}

Received: 20 November 2018/Revised: 14 December 2018/Accepted: 14 December 2018/Published online: 3 January 2019

(C) Canadian Anesthesiologists' Society 2019

A 48-yr-old man was scheduled for dental extractions under general anesthesia. He had been on rivaroxaban for ten months (having been on warfarin for the prior three decades) because of multiple episodes of deep vein thrombosis (DVT) and pulmonary embolism. These episodes had occurred despite continuous anticoagulation and use of anti-embolic stockings. Physical examination revealed lower limb congestion with pitting edema, venous varicosities, stasis dermatitis, skin ulceration (Figure A), as well as some dilated and thrombosed superficial abdominal veins.

A computed tomography scan of the abdomen four years earlier (to investigate enlarged inguinal lymph nodes found on a venous Doppler scan in the setting of lower limb cellulitis) incidentally revealed the absence of the inferior vena cava (IVC). This was attributed to a congenital anomaly due to radiologic (isolated absence of the infrarenal IVC segment, with preserved suprarenal IVC

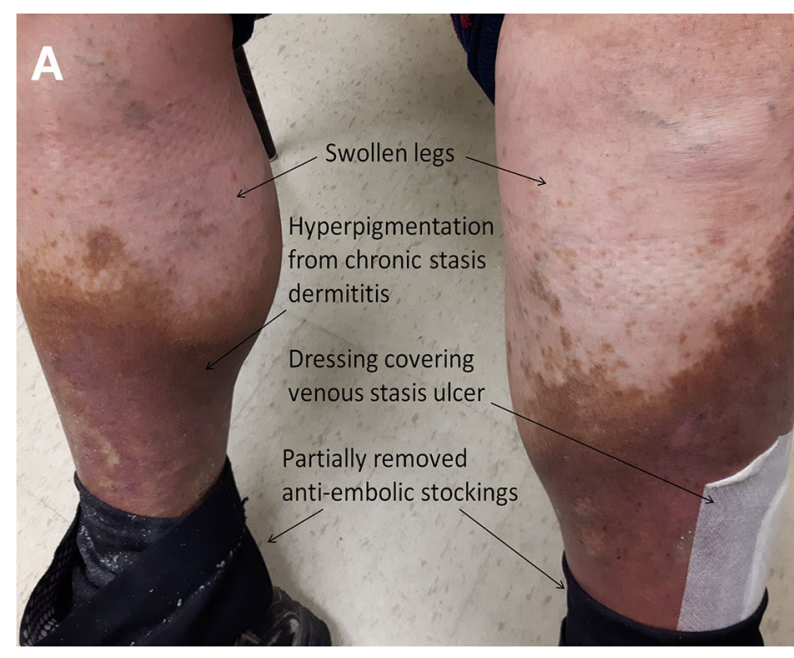

Figure A) Photograph of the patient with congenital absence of the inferior vena cava (IVC) shows edema, stasis dermatitis, and trophic skin changes resulting from chronic venous congestion. B) Coronal delayed-phase (with contrast) computed tomography of the abdomen shows the absence of the IVC, with retroperitoneal collateral vessels

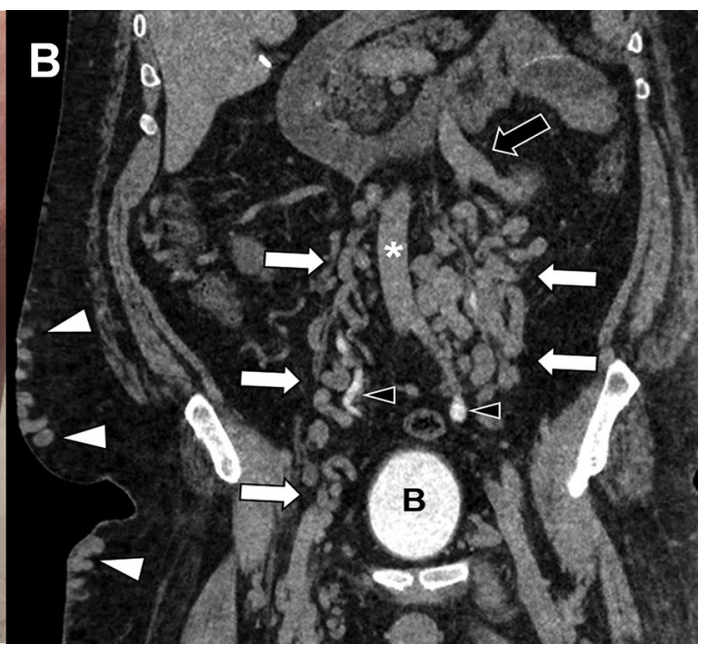

(white arrows) surrounding the abdominal aorta (asterisk) and the opacified ureters (black arrowheads). The inferior mesenteric vein (black arrow) is dilated, related to collateralization with systemic venous drainage to the portal venous system. Superficial collateral vessels are also noted (white arrowheads). B = urinary bladder

A. M.-H. Ho, MD, FRCPC, FCCP .

G. B. Mizubuti, MSc, MD ( $\triangle)$

Department of Anesthesiology and Perioperative Medicine,

Queen's University, Kingston, ON, Canada

e-mail: gleniomizubuti@hotmail.com

A. D. Chung, MD, FRCPC

Department of Radiology, Queen's University, Kingston,

ON, Canada 
and iliac veins) and clinical (previous unexplained DVT in a $<30$-yr-old male) ${ }^{1}$ characteristics (Figure). As dental extractions can be performed in anticoagulated patients, ${ }^{2}$ we proceeded without stopping his rivaroxaban. His perioperative course was uneventful.

This rare condition may be present in up to $5 \%$ of young people with otherwise unexplained DVT. ${ }^{3}$ Without an IVC, venous return generally relies on dilated and stasis/ insufficiency-prone collateral vessels. These patients have a further increased risk of DVT in association with cancer, surgery, smoking, hormonal therapy, heart failure, pelvic obstruction (neoplasia/abscess/hematoma/pregnancy), increased intra-abdominal/intrathoracic pressure, and/or prolonged immobility. Strenuous exercise increases lower limb blood flow and may exacerbate venous congestion.

Perioperatively, intravenous cannulation in the lower limb for infusion (risk of increased thrombosis potential) and/or pressure monitoring (additional venous obstruction and unreliable measurements) should be discouraged; ultrasound-based assessment of the IVC to guide fluid therapy is impossible; and the engorged epidural/paravertebral veins (Figure B) may result in increased bleeding risk during spine surgery and/or lumbar puncture/ neuraxial/paravertebral anesthesia techniques. Bleeding risk may increase during certain abdominal surgery. Perioperative anticoagulation and anti-fibrinolytic management must balance the risk of bleeding versus thrombosis.
Conflicts of interest None declared.

Editorial responsibility This submission was handled by Dr. Hilary P. Grocott, Editor-in-Chief, Canadian Journal of Anesthesia.

Consent Patient signed an informed consent for publication of this case. In addition, institutional ethical approval was obtained as required for the current work.

Funding Support was provided solely from institutional and/or departmental sources. No external funding was acquired for completion of the current work.

\section{References}

1. Lambert M, Marboeuf $P$, Midulla $M$, et al. Inferior vena cava agenesis and deep vein thrombosis: 10 patients and review of the literature. Vasc Med 2010; 15: 451-9.

2. Weltman NJ, Al-Attar $Y$, Cheung $J$, et al. Management of dental extractions in patients taking warfarin as anticoagulant treatment: a systematic review. J Can Dent Assoc 2015; 81: f20.

3. O'Connor DB, O'Brien N, Khani T, Sheehan S. Superficial and deep vein thrombosis associated with congenital absence of the infrahepatic inferior vena cava in a young male patient. Ann Vasc Surg 2011; 25: 697.e1-4.

Publisher's Note Springer Nature remains neutral with regard to jurisdictional claims in published maps and institutional affiliations. 